\title{
An equivalent dissipation rate model for capturing history effects in non-premixed flames
}

\author{
Prithwish Kundu ${ }^{\mathrm{a}, \mathrm{b}, *}$, Tarek Echekki $^{\mathrm{a}}$, Yuanjiang Pei ${ }^{\mathrm{b}}$, Sibendu Som ${ }^{\mathrm{b}}$ \\ ${ }^{a}$ Department of Mechanical and Aerospace Engineering, North Carolina State University, Raleigh, NC 27695, USA \\ ${ }^{b}$ Energy System Division, Argonne National Laboratory, Lemont, IL, USA
}

\begin{abstract}
The effects of strain rate history on turbulent flames have been studied in the past decades with 1D counter flow diffusion flame (CFDF) configurations subjected to oscillating strain rates. In this work, these unsteady effects are studied for complex hydrocarbon fuel surrogates at engine relevant conditions with unsteady strain rates experienced by flamelets in a typical spray flame. Tabulated combustion models are based on a steady scalar dissipation rate (SDR) assumption and hence cannot capture these unsteady strain effects; even though they can capture the unsteady chemistry. In this work, 1D CFDF with varying strain rates are simulated using two different modeling approaches: steady SDR assumption and unsteady flamelet model. Comparative studies show that the history effects due to unsteady SDR are directly proportional to the temporal gradient of the SDR. A new equivalent SDR model based on the history of a flamelet is proposed. An averaging procedure is constructed such that the most recent histories are given higher weights. This equivalent SDR is then used with the steady SDR assumption in 1D flamelets. Results show a good agreement between tabulated flamelet solution and the unsteady flamelet results. This equivalent SDR concept is further implemented and compared against 3D spray flames (Engine Combustion Network Spray A). Tabulated models based on steady SDR assumption under-predict autoignition and flame lift-off when compared with an unsteady Representative Interactive Flamelet (RIF) model. However, equivalent SDR model coupled with the tabulated model predicted autoignition and flame lift-off very close to those reported by the RIF model. This model is further validated for a range of injection pressures for Spray A flames. The new modeling framework now enables tabulated models with significantly lower computational cost to account for unsteady history effects.
\end{abstract}

Keywords: Flamelet History, Engine Combustion Network, Tabulation

\section{Introduction}

Turbulent non-premixed flames are subject to unsteady strain effects, also referred to as history effects. The influence of unsteady strain on flames with respect to ignition, extinction, and species concentration has been the focus of numerical and experimental work by many researchers in the past decades. Peters and William [1] discussed a flame stabilization mechanism for non-premixed counter flow diffusion flames (CFDF) based on quenching limits of

${ }^{*}$ Corresponding author

Email address: pkundu@ncsu.edu (Prithwish Kundu) 
flamelets and their dependence on scalar dissipation rate (SDR). This was also supported by findings of Mastorakos et al. 2. Egolfopoulos et al. 3] numerically studied 1D CFDF with periodic strain rates. The flame response was quasi-steady for very high and very low frequencies. However, the intermediate frequencies showed a phase shift between the oscillations and the flame response. Similarly, Kistler et al. [4] carried out experimental and numerical study of CFDFs and observed that for very low and high frequencies in strain oscillations, the behavior was quasisteady. Extinction was not observed for very high frequencies with peak strain values beyond quenching limits. Im et al. 5] studied CFDFs under oscillating strain with similar conclusions. It was suggested that as the strain rate increases beyond the extinction limits, the flamelet needs some time to respond to this rapid change. If the time scale of oscillation is not long enough then these high strain rates are not sufficient for the flame to extinguish. Similar results were observed by Brown et al. [6]. Barlow et al. [7] studied the effect of a temporal step change (sudden decrease) of strain on flamelets experimentally as well as numerically using the steady flamelet assumption. The results showed that the steady flamelet assumption over-predicted the $\mathrm{OH}$ and $\mathrm{CO}$ species concentrations. Overall, these studies show that the flame response, including its ignition characteristics depends not only on scalar dissipation rate, but also on its history.

The Representative Interactive Flamelet (RIF) model solves the unsteady flamelet equations at each time-step, thus accounting for the history effects in flamelets. These models have been implemented in a number of simulations over a wide range of spray combustion regimes from HCCI (homogeneous charge compression ignition) to diesel combustion [8, 9, 10, 11, 12, 13, 14, 15, 16. However, the need for multiple flamelets and the online solution of flamelet equations at each time step have resulted in these models being computationally expensive and prohibitive for high-fidelity engine simulations. A less expensive method is to solve the flamelet equations a priori, for a range of conditions and tabulate the species as a lower dimensional manifold. The unsteady nature of chemistry is accounted in these manifolds through the implementation of a progress variable. This category of tabulated flamelet models include Flamelet Progress Variable (FPV) [17, 18, Unsteady Flamelet Progress Variable (UFPV) [19, 20, 21] and Flamelet Generated Manifolds (FGM) 22, 23, 24, 25].

Large chemistry mechanisms necessary for accurate simulation of hydrocarbon fuels lead to high computational costs. Efforts have been directed towards speeding up stiff chemical kinetics calculations [26]. Tabulated flamelet models have been used extensively and have successfully reduced computational costs in practical combustion problems while incorporating high fidelity chemistry mechanisms. The progress-variable type unsteady models, which can take into account unsteady chemical kinetics, however, cannot account for the effect of unsteady strain rate history. The lower dimensional manifolds are generated for a range of SDRs by solving the flamelet equations. During these computations the SDR of each flamelet equation is kept constant. This is referred to as the steady strain assumption and implies that the flamelet can instantaneously adjust to the local scalar dissipation rate. As a result, the resulting model cannot capture the effect of unsteady strain rate history of a flamelet. Various approaches that attempt to incorporate unsteady effects in recent years have been primarily restricted to oscillatory strain for flames under 
atmospheric pressures. Haworth et al. 27] studied the effects of time varying strain rates on flamelets and suggested a formulation to calculate an equivalent strain based on the history of the strain rate. The equivalent strain was then coupled with a tabulated steady flamelet library. It needs to be investigated how these methods can be applied towards unsteady tabulated flamelet libraries. Cuenot et al. 28, proposed the idea of calculating an equivalent strain based on the history of a flamelet. This formulation and its validation was based on single-step chemistry and a single time scale associated with the frequency of strain rate oscillations. For chemistry involving multiple species, an equivalent strain needs to be calculated for each specie. Delhaye et al. 29] developed a framework to incorporate unsteady effects in FGM based on 2D (with 2 controlling variables) and 3D (with 3 controlling variables) manifold. This was used to predict species for a flamelet subject to periodic strain rate and compared with detailed unsteady simulations using GRI 3.0 chemistry mechanism [30] for methane. The 3D manifold resulted in better comparisons with the unsteady periodic strain rate simulation. In contrast to the 2D manifold, this did not exhibit a phase shift relative to the unteady simulations. The work was further extended to extinction limits by Delhaye et al. [31].

Studies based on periodic strain rate oscillations may be relevant to a number of applications. However, these findings cannot be generalized to other configurations, such as the configuration of interest here, which corresponds to spray flames under diesel-like conditions [14, 15. More importantly, it is essential to capture the onset of autoignition and the transition to lifted flames. A strong correlation between these parameters and pollutant formation have been established for diesel flames. The scalar dissipation rates in spray flames experience much larger gradients and decay exponentially over a short period of time, as the flamelet like structures move away from the nozzle, as shown in previous studies by our group [14. Understanding the unsteady history effects in these flames that lead to the onset of autoignition or extinction behavior is a principal motivation for this work. More importantly, the ability to exploit a tabulation scheme for these effects as an alternative strategy to in situ unsteady flamelet simulations may provide a significant computational saving, given the chemistry complexity associated with practical fuels.

The objectives of this work are two-fold. The first objective is to quantify these history effects and to investigate if these effects are significant for diesel injection applications. The second objective is to develop a model that can incorporate these effects, and implement a tabulation approach for these effects to overcome the inherent computational cost of in situ unsteady flamelet simulations. Strain rates in flamelet computations are best represented through an equivalent contribution in mixture fraction space, the SDR, which is normally identified with the dissipation rate at stoichiometric mixture conditions. In the following sections, we first attempt to quantify the contribution of dissipation rate and its temporal variations on the autoignition process and subsequent high-temperature combustion (Sec. 2). Then we propose an equivalent dissipation rate model that is designed to capture dissipation rate histories as presented in Sec. 2.4. Finally, this concept is validated for 1D flamelet calculations (Sec. 3), and 3D RANS simulations are presented and discussed in Sec. 4. Conclusions are presented in Sec. 5. 


\section{Evaluation of history effects in 1D flamelets under diesel-relevant conditions}

To understand the role of unsteady dissipation rates on the evolution on autoignition and the formation of non-premixed flamelets, 1D unsteady flamelet simulations are carried out. The same simulations will provide the database to construct the equivalent dissipation rate model discussed below. The flamelet problem is set up for high pressure engine relevant conditions with n-dodecane as the fuel surrogate for diesel. The pressure is set to 60 bar and oxidizer stream is diluted with $\mathrm{CO}_{2}$ and $\mathrm{H}_{2} \mathrm{O}$ as per engine relevant Sandia Spray A exhaust gas re-circulation (EGR) conditions [32]. The ambient oxidizer temperature is set at $900 \mathrm{~K}$. The stoichiometric scalar dissipation rate for the flamelet is varied linearly over time in the first part of the 1D study. The flamelets are initialized at unburnt conditions and the unsteady igniting flamelet problem is solved up to $0.63 \mathrm{~ms}$. The 106 species n-dodecane chemistry mechanism with 420 reactions is used to model the chemical kinetics in the unsteady flamelet and all the other 3D CFD simulations 33 .

In the section below, we study a $1 \mathrm{D}$ counter-flow diffusion flame subject to time-varying scalar dissipation rates with 3 different modeling approaches. They include 1) the unsteady flamelet model, which is the most accurate, and also the most expensive method for determining the effects of time-evolving dissipation rates, 2) the steady SDR flamelet approach, which looks up the solution of the current SDR without accounting for its time history, and 3) the equivalent SDR model, which is proposed within the context of the present work.

\subsection{Unsteady flamelet model}

In this model,the following unsteady flamelet equations are solved:

$$
\rho \frac{\partial Y_{i}}{\partial t}=\rho \frac{\chi}{2} \frac{\partial^{2} Y_{i}}{\partial Z^{2}}+\dot{\omega}_{i}
$$

where $\chi=\chi_{s t} \frac{f(Z)}{f\left(Z_{s t}\right)}$ and $f(Z)=\exp \left[-2\left(\operatorname{erfc} c^{-1}(2 Z)\right)^{2}\right]$

$$
\begin{array}{r}
\rho \frac{\partial T}{\partial t}-\rho \frac{\chi}{2} \frac{\partial^{2} T}{\partial Z^{2}}-\rho \frac{\chi}{2 C_{p}}\left[\sum\left(\frac{C_{p i}}{L e_{i}} \frac{\partial Y_{i}}{\partial Z}\right)+\frac{\partial C_{p}}{\partial Z}\right] \frac{\partial T}{\partial Z}= \\
\frac{1}{C_{p}}\left(\frac{\partial P}{\partial t}-\sum \dot{\omega}_{i} h_{i}\right)
\end{array}
$$

In these equations, $Y_{i}$ is the species mass fraction, $\rho$ is the density, $\omega_{i}$ is the species source term. $C_{p}$ is the specific heat at constant pressure, $P$ is the pressure, $h$ is the enthalpy and $\chi$ is the scalar dissipation rate. Unity Lewis $L e_{i}$ number was assumed in this study. At each time-step, the stoichiometric scalar dissipation rate changes and is updated for the flamelet equations. Thus, this numerical model is capable of incorporating unsteady strain effects, i.e., the history of the strain rate is accounted for in the model, and ignition of flamelets will be dependent on the previous history of the flamelet. This modeling approach is the equivalent of the RIF model in 1D. 


\subsection{The steady SDR flamelet assumption}

A 1D flamelet model, which mimics the solution retrieval from a tabulated manifold, is developed in this section. Tabulated flamelet libraries are generated a priori for a range of stoichiometric SDRs. This constant SDR assumption neglects history effects. In order to mimic the solution from a tabulated method, the following scheme is implemented in the 1D flamelet solver. The flamelets are first initialized to unburnt conditions. The scalar dissipation rate is then updated, which is a function of time. The partial differential equations (PDE) are now solved from time $t=0$ up to the current time step $t_{1}$. The flamelet solution is finally available for time $t_{1}$. The stoichiometric scalar dissipation rate changes with time. For the solution at second time step, $t_{2}$, the flamelet is again reinitialized to the unburnt condition and the PDEs are solved from time $t=0$ to $t_{2}$ with the new value of scalar dissipation rate. The solutions are then stored for time $t_{2}$. This is done iteratively for the entire simulation time. Thus, the solution at each time step is independent of the previous time step and a function of the SDR at the current time step. This eliminates the history effects of the temporally changing SDR. It is important to note that this procedure eliminates only the unsteady effects of the changing scalar dissipation rate. The unsteadiness related to chemical kinetics is preserved. This numerical procedure mimics the solution retrieval from a tabulated manifold, with unsteady chemistry, but without incorporating interpolation and tabulation errors. This enables a more consistent comparison with the other models.

\subsection{Evaluation of history effects in flamelets}

Figure 1 shows the temporal variations in stoichiometric scalar dissipation rates experienced by different flamelets in a spray flame [14]. The multiple flamelet RIF model consists of injecting the multiple flamelets sequentially based on injected fuel mass. Consider a $10 \mathrm{mg}$ fuel spray injection event modeled using 10 flamelets. The first flamelet is initialized and transported during the first $1 \mathrm{mg}$ of fuel injection. The second flamelet is initialized at the end of $1 \mathrm{mg}$ of fuel injection. This process is continued for the entire injection duration with a new flamelet being initialized in succession. The values presented for the SDRs and their rate of variations are consistent with the experimental conditions of interest, which correspond to ECN Spray A. These dissipation rates vary from $0 \mathrm{~s}^{-1}$ to $380 \mathrm{~s}^{-1}$. At the start of spray, the stoichiometric regions are close to the spray region and hence experience very high $\chi_{s t}$. As these regions move away from the spray regions, $\chi_{s t}$ decreases sharply and then reaches a steady state. Similar observations were reported by D'Errico et al. [15. History effects due to such large temporal gradients need to be evaluated for diesel engine conditions. In this part of the study, flamelets under high temperature and pressure conditions are subjected to varying SDRs and evaluated using the two models described in the previous sections. The differences between the results from the two models demonstrate the influence of history effects on an igniting flamelet. In order to study the effect of varying SDRs systematically, the flamelet problems are first studied with uniformly varying SDR, i.e., constant $\frac{d \chi_{s t}}{d t}$. The result of such variations on flamelets are shown in Fig. 2 ,

Figure 2 shows the maximum temperature as a function of time for the flamelets and their stoichiometric scalar

dissipation rates on the secondary $y$-axis. It is observed that for a low gradient case, $\left(\frac{d \chi_{s t}}{d t}=2.3 \times 10^{4}\right)$ the ignition 
observed from the unsteady RIF-1D equations is close to the predictions from the steady strain model. Thus, the history effects do not have an impact on autoignition under such conditions where the $\chi_{s t}$ in a flamelet is changing slowly and the quasi steady assumption is valid. However, as the rate of change of SDR is increased, the difference between ignition predicted by the RIF model and steady strain assumption keep increasing. As the SDR decreases at a faster rate, the flamelet needs some time to adjust to the lower $\chi_{s t}$, as also observed in previous studies [7.

The RIF model is sensitive to these history effects and ignites at a later time step compared to the steady strain assumption. The ignition delay as a function of $\frac{d \chi_{s t}}{d t}$ is summarized in Fig. 3 . The ignition delay for each flamelet was defined as the time at which the maximum gradient of maximum temperature occurs. Thus, it is clear that the difference in autoignition of flamelets due to history effects is proportional to the magnitude of the temporal gradient in SDR experienced by the flamelet. These 1D results also show that autoignition in diesel engine conditions are sensitive to the history of the scalar dissipation rates and there is a need to account for such effects. Tabulated models operating under the steady SDR assumption will predict a different ignition delay compared to the more accurate in situ models like RIF.

\subsection{The Equivalent SDR model}

The two approaches that are already discussed above have positive attributes with respect to implementation on a CFD solver. The unsteady flamelet approach (RIF) is more accurate; but it must be implemented in situ, which makes the incorporation computationally expensive. The added computational cost is compounded with the cost of integrating complex fuels' chemistry. The second approach of tabulating a manifold is computationally more efficient. However, it does not incorporate history effects, which affects among other measures, the onset of autoignition and the overall combustion process. The aim of this model is to incorporate the effect of unsteady SDR in a tabulated flamelet framework. For a given unsteady flamelet problem, the history of the scalar dissipation rate is known up to the current time step. Based on the previous history, an equivalent SDR is calculated using an averaging procedure. This equivalent SDR accounts for the unsteady SDRs experienced by the flamelet. The basic premise of the proposed equivalent SDR model is that the most recent histories of the flamelet will contribute more to the current solution of the flamelet than its older histories. A weight function is implemented for this averaging procedure, which can be expressed as follows:

$$
w(t)=\frac{e^{20 t}}{e^{20 c}+e^{20 t}}
$$

The limits of the weight function are from 0 to 1 as required for the averaging procedure and $c$ is a parameter that controls the distance of the step from $t=0$ as shown in Fig 4 .

An equivalent SDR is calculated using weight averaging for a flamelet with varying SDR. Figure 4 shows the temporal variation of SDR corresponding to Spray A flame, plotted against non-dimensional time [14]. The figure also illustrates the shape of the proposed weight function, which represents a function that gradually transitions from a value of 0 (no influence) to 1 (most influence). The current time since the start of the flamelet initialization is used 
to normalize time; thus, $t=1$ represents the current time. At each time step of the flamelet calculation, the history of the scalar dissipation rate $\chi_{s t}(t)$ is known. The equivalent SDR at each time step is calculated using the following equation:

$$
\chi_{c}=\frac{\int \chi_{s t}(t) w(t) d t}{\int w(t) d t}
$$

This equivalent SDR is then used as the stoichiometric SDR for the flamelet. This model works on the approach similar to that of the steady SDR model approach where the SDR is replaced by an equivalent SDR. Consider an example where the parameter $c=0.7$. When the equivalent SDR is calculated using Eq. (4), the SDRs corresponding to the normalized time greater than 0.7 will contribute more towards the average. The SDRs corresponding to normalized time less than 0.7 will gradually contribute less to the average. Thus, by implementing the step function, the most recent history will contribute more to the equivalent SDR. This process can be controlled by the variation of $c$. Smaller values of $c$ will shift the step function to the left and result in more time averaging and vice-versa. This equivalent SDR is then used in the steady SDR 1D model described in the previous section. The following sections show the effect of this model and the influence of the correction factor $c$.

\section{The Equivalent SDR model - 1D}

To demonstrate the effects of the equivalent SDR model on the flamelet response and autoignition delay, the flamelets are subjected to different temporal gradients $\left(d \chi_{s t} / d t\right)$ in scalar dissipation rates like the previous study [14. The equivalent SDR model is applied to the steady strain assumption described in the previous section. The equivalent SDR model calculates an averaged $\chi_{\text {st }}$ at each time step and couples it with the steady SDR assumption model. Parametric variations in $c$ are reported on the first sub-figure on the left in Fig. 5. It is observed that the use of the equivalent SDR shifts the maximum temperature curve towards the unsteady results. For smaller gradients, higher values of $c$ are enough to predict the unsteady results and vice versa, i.e., more time averaging is required for higher gradients. Clearly, the optimum value of $c$ depends on $d \chi_{s t} / d t$. These sets of $1 \mathrm{D}$ flamelet simulations with different gradients are used to construct a correlation between $d \chi_{s t} / d t$ and the optimum $c$. This correlation enables the equivalent SDR model to be independent of tuning parameters and the correction factor $c$ is calculated dynamically at each time step for cases where $\chi_{s t}$ varies non-linearly.

\subsection{Validation of equivalent SDR concept for non uniformly varying SDRs in flamelets}

The previous sections discussed application of the equivalent SDR concept for cases where the SDRs were varying linearly. However, as shown in Fig. 11 the change in SDR experienced by flamelets in spray flames under engine conditions are non-linear. The temporal gradient changes continuously at each time step. In this section, the variation of SDR from a spray flame is imposed on a 1D flamelet as shown in Fig. 6. This temporal variation in $\chi_{\text {st }}$ is obtained from one of the RIF flamelets from the Spray A simulation in [14]. The conditions are the same as Spray 
A flame condition with ambient $\mathrm{O}_{2}$ concentration of $15 \%$, ambient pressure of 60 bar and ambient temperature of $900 \mathrm{~K}$. This flamelet problem is solved using the 3 different models discussed in the previous sections: 1) Steady SDR assumption, which is equivalent to a tabulated manifold, 2) Unsteady Flamelet model, which is equivalent to 1D RIF, 3) Equivalent SDR (ES) concept. At each time step, the value of $\chi_{s t}$ is imposed from a Spray A flame simulation. For the ES model, the optimum value of correction factor $c$ is calculated, at each time step, based on the correlation developed in the previous section between $c$ and $d \chi_{s t} / d t$ based on a linear temporal profile for the SDR. Figure 6 shows the ignition of the flamelet under these conditions with different models. The flamelet with the steady strain assumption predicts the first stage ignition at $0.1 \mathrm{~ms}$ followed by the main ignition at $0.3 \mathrm{~ms}$. The unsteady model on the other hand predicts the first stage ignition at $0.2 \mathrm{~ms}$ and then the main ignition at $0.5 \mathrm{~ms}$. Thus, it is clear that under engine conditions, for spray flames, the history effects in flamelets have a strong influence on autoignition. The ignition depends on the coupled effects of SDR and chemistry. As $\chi_{s t}$ decreases rapidly beyond the quenching limit, the species formation reactions proceed leading to heat release. An accurate solution would need to integrate the chemistry over time as per the varying SDRs, i.e., the procedure followed by the unsteady RIF model. In tabulated models, a lower dimensional manifold is generated a priori for a set of values of $\chi_{s t}$. The solution at each point is retrieved from the manifold based on $\chi_{s t}$ independent of its history, i.e., the steady SDR approach. On the other hand, the first stage and main ignition from the ES model, in Fig. 6 6 matches the RIF model predictions reasonably well. Thus the ES approach is shown to be capable of predicting ignition of flamelets in tune with the RIF model for arbitrarily chanding SDRs.

The results from the studies so far show that history effects have a significant impact on the prediction of autoignition in diesel engine-type applications. This has been demonstrated in flamelets with non-linearly varying SDRs. Also, the ES concept when coupled with steady strain assumption can predict ignition delays close to the ones predicted by in situ solution of flamelet equations. These results have implications towards computational fluid dynamics simulations. If the equivalent SDR concept is applied to tabulated combustion models in CFD, the resulting models will be able to incorporate history effects at significantly lower computational costs compared to the RIF models.

The 1D flamelet setup with the new model were solved for the same gradients as shown in Fig. 2 but for different magnitudes of scalar dissipation rates. In the previous studies the final SDR at the end of $0.63 \mathrm{~ms}$ was set as 10 $\mathrm{s}^{-1}$. In these tests, the final SDR is set to $20 \mathrm{~s}^{-1}$. The results shown in Fig. 7 reveal that the optimum value of $c$ is also dependent on $\chi_{s t}$. Therefore, it is a function of both $\chi_{s t}$ and $d \chi_{s t} / d t$ for a given mixture condition and can be tabulated accordingly in terms of these 2 parameters. In order to implement a predictive CFD model without parameter tuning, a scheme is developed that calculates this correction factor $c$ based on $\mathrm{d} \chi_{s t} / \mathrm{dt}$ and $\chi_{s t}$. i.e. $c=f\left(\chi_{s t}, \mathrm{~d} \chi_{s t} / \mathrm{dt}\right)$. In order to achieve this, a number of 1D flamelet equations are solved iteratively for different values of $c$ for a given value of $d \chi_{s t} / d t$ and $\chi_{s t}$. The value of $c$ which predicts the autoignition closest to the unsteady results are selected and tabulated in a 2D table. This secondary $2 \mathrm{D}$ table represents the correction factor 
as a function of $\mathrm{d} \chi_{s t} / \mathrm{dt}$ and $\chi_{s t}$.

\section{Equivalent SDR Model for 3D CFD simulations}

The equivalent SDR concept incorporates unsteady effects in flamelets using steady SDR flamelet libraries. This concept is implemented and validated for 3D non-premixed turbulent spray flame CFD simulations. Two novel modeling approaches are implemented, 1) The Tabulated Flamelet Model (TFM), based on the steady strain assumption, and, 2) The Tabulated Equivalent Strain Flamelet model (TESF) based on the equivalent SDR concept developed in the current study. The conditions of Spray A flame mimic the ambient conditions encountered in typical diesel engines. The experimental setup consists of a constant-volume combustion chamber in which the ignition of a premixed fuel-air mixture 34 is used to achieve the target ambient conditions. Once the desired conditions are reached the fuel is injected into the constant-volume chamber through a single-hole injector. The computational domain that mimics the experiment is shown in Fig. 8 .

The tabulated combustion models developed in this work were coupled to the CONVERGE CFD code (2.1 version) [35. It uses an innovative cut-cell method with the capability to generate meshes at run-time. The 3D grid can be refined at run-time based on the physics of the problem. This is achieved using adaptive mesh refinement. The gas phase is treated as a continious Eulerian phase and the liquid spay is treated as discrete Lagrangian parcels. The turbulent flow-field is modeled using the renormalization group (RNG) Reynolds Average Navier-Stokes (RANS) based turbulence model [36]. The spray injection is modeled using the blob injection approach [37. The KevinHelmholtz (KH) [38] and the Rayeigh-Taylor(RT) [39] models are employed to model the secondary breakup process. The Frossling correlation [40] is used for modeling droplet evaporation. A dynamic drag model and a turbulent dispersion model is used to model drag and dispersion [41. The combustion models developed were coupled with the CFD flow solver and can be extended to Large Eddy Simulation (LES) turbulence models as well. The integration of the combustion models are shown schematically in Fig. 9. The 106 species and 420 reactions, n-dodecane chemistry mechanism has been used for all the models 33 .

\subsection{Representative Interactive Flamelet model (RIF)}

The multi-flamelet RIF model and the spray models have been extensively used and validated for spray simulations over a wide range of conditions in our previous study [14]. In this model, the unsteady flamelet equations are solved for each flamelet at each time step. This accounts for the history effects in the flamelets. The online flamelet libraries are computationally expensive. This motivates the development of tabulated models capable of incorporating unsteady effects.

The following equations are solved by the CFD solver: continuity, momentum, energy, mixture fraction and mixture fraction variance. Each iteration of the CFD solver calculates the mixture fraction and enthalpy values for 
each cell. The mixture fraction and mixture fraction variance transport equations are shown in Eqs. 5 - 7.

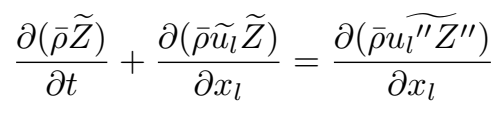

where

$$
\begin{gathered}
\widetilde{u_{l}^{\prime \prime} Z^{\prime \prime}}=-D_{t} \frac{\partial \tilde{Z}}{\partial x_{l}} \\
\frac{\partial\left(\widetilde{\rho} \widetilde{Z^{\prime \prime}}\right)}{\partial t}+\frac{\partial\left(\bar{\rho} \widetilde{u_{l}} \widetilde{Z^{\prime \prime}}\right)}{\partial x_{l}}=-\frac{\partial\left(\bar{\rho} u_{l}^{\prime \prime Z^{\prime \prime}}\right)}{\partial x_{l}}-2\left(\bar{\rho} \widetilde{u_{l}^{\prime \prime} Z^{\prime \prime}}\right) \frac{\partial \widetilde{Z}}{\partial x_{l}}-\bar{\rho} \widetilde{\chi}
\end{gathered}
$$

The flamelet equations are then solved for the given time step and its solution gives the species mass fractions as a function of mixture fraction $Y(Z)$ for each flamelet. The flamelet equations are shown in Eqs. 1 and 2 Equation 1 is obtained by a coordinate transformation of the species transport equation to mixture fraction space. Similar coordinate transformation of the energy conservation equation gives Eq. (2). These two flamelet equations represent the unsteady chemistry in mixture fraction space. The scalar dissipation rate is modeled as shown in Eq. (8) where $c_{x}$ is a mixing constant, $k$ is the turbulent kinetic energy, and $\varepsilon$ is the dissipation rate obtained from the turbulence model. The value of $c_{x}$ was set equal to 2 for all cases. The CFD rtesults are not sensitive to $c_{x}$.

$$
\widetilde{\chi}=c_{x} \frac{\varepsilon}{k} \widetilde{Z^{\prime 2}}
$$

In order to solve these set of PDEs, Eqs.1 and 2, the scalar dissipation rate $\chi$ is converted from a function of physical space to a function of mixture fraction. The following equation derived in [42] is used to perform the transformation:

$$
\chi(Z)=\widehat{\chi_{s t}} \frac{f(Z)}{f\left(Z_{s t}\right)}
$$

where

$$
f(Z)=\exp \left[-2\left(e r f c^{-1}(2 Z)\right)\right]
$$

$\widetilde{\chi_{s t}}$ is the domain averaged scalar dissipation rate conditional over the stoichiometric mixture fraction and erfc is the complimentary error function. In order to construct the flamelet library for the entire CFD domain, the average value is calculated using Eq. (11):

$$
\widehat{\chi_{s t}}=\frac{\int_{v} \bar{\rho} \widetilde{\chi_{s t}}{ }^{3 / 2} \widetilde{P}\left(Z_{s t}\right)}{\int_{v} \bar{\rho} \widetilde{\chi_{s t}}{ }^{1 / 2} \widetilde{P}\left(Z_{s t}\right)}
$$

where

$$
\widetilde{\chi_{s t}}=\widetilde{\chi} \frac{f\left(Z_{s t}\right)}{\int_{0}^{1} f(Z) P(Z) d Z}
$$


$v$ is the volume of the entire computational domain and $P()$ is the probability density function.

A $\beta$-PDF is constructed using the mean and variance $(\widetilde{Z " 2})$ of the mass fractions as shown in Eq. 13 , where, $\Gamma$ is the gamma function. This PDF is multiplied by the species function $Y(Z)$ and then integrated to give the final species mass fraction (Eq. (14)). Once the species mass fractions are calculated at each cell, the temperatures can be back-calculated from the enthalpy. This process is continued iteratively for the entire simulation.

$$
P(Z ; \boldsymbol{x}, t)=\frac{\widetilde{Z}^{\alpha-1}(1-\widetilde{Z})^{\beta-1}}{\Gamma(\alpha) \Gamma(\beta)} \Gamma(\alpha+\beta)
$$

where $\alpha=\tilde{Z} \gamma ; \quad \beta=(1-\tilde{Z}) \gamma \quad$ and $\quad \gamma=\frac{\widetilde{Z}(1-\widetilde{Z})}{\widetilde{Z^{\prime \prime 2}}}-1$

$$
\tilde{Y}_{i}(\boldsymbol{x}, t)=\int_{0}^{1} Y_{i}(Z, t) P(Z ; x, t) d Z
$$

\subsubsection{Multiple Flamelets}

The scalar dissipation rate calculated in Eq. [11 is an average value over the entire computational domain. In applications like turbulent spray flames, the scalar dissipation rate can vary by an order of magnitude in space as shown in Fig[1. The averaging process, therefore, can introduce large errors. Barths et al. [9] suggested the use of multiple flamelets with each flamelet having its own $\chi_{s t}$. As per this approach, the fuel injection is divided over multiple flamelets serially and each flamelet's chemistry is governed by a set of flamelet equations. The different flamelets are tracked using marker equations shown in Eq. [15], which are solved for each flamelet.

$$
\frac{\partial\left(\bar{\rho} \widetilde{Z}_{l}\right)}{\partial t}+\frac{\partial\left(\bar{\rho}{\widetilde{u_{l}}}_{Z_{l}}\right)}{\partial x_{l}}=-\frac{\partial\left(\bar{\rho} u_{l} Z_{l}^{\prime \prime}\right)}{\partial\left(x_{l}\right)}
$$

These markers must also satisfy the following equation:

$$
\widetilde{Z}=\sum_{l=1}^{n} \widetilde{Z}_{l}
$$

where $n=$ number of flamelets. The averaged value of stoichiometric scalar dissipation rate for each flamelet $(l)$ is calculated as:

$$
\widehat{\chi_{s t}}(l)=\widehat{\chi_{s t}}=\frac{\int_{v} \frac{\widetilde{Z_{l}}}{Z} \widetilde{\rho}_{\chi_{s t}}^{3 / 2} \widetilde{P}\left(Z_{s t}\right)}{\int_{v}{\widetilde{Z_{l}}}_{Z} \bar{\rho}{\widetilde{\chi_{s t}}}^{1 / 2} \widetilde{P}\left(Z_{s t}\right)}
$$

The final species mass fraction at each cell is calculated by taking a weighted average of species mass fractions obtained from all flamelets as shown in Eq. 18 .

$$
\widetilde{Y}_{i}=\sum_{l=1}^{n} \frac{\widetilde{Z}_{l}}{\widetilde{Z}} \widetilde{Y}_{l}
$$




\subsection{Tabulated flamelet model (TFM)}

This novel tabulation technique is based on the framework of multi-flamelet RIF model by replacing the in situ flamelet solver with a tabulation approach. The flamelet equations are solved before run time for a range of $\chi_{s t}$, mixture fraction $Z$, mixture fraction variance $Z^{\prime \prime 2}$ and time $t$. This generates a 4 dimensional flamelet library with time as one of the independent variables. In this flamelet library, the species mass fractions are tabulated as functions of these 4 independent variables. A $\beta$-PDF is used to account for the turbulence-chemistry interaction effects. This method of tabulation does not use progress variables to model the unsteadiness in chemical kinetics. The $4 \mathrm{D}$ manifolds are an ensemble of different 3D manifolds and hence this problem can be parallelized without the need for communication between the processors. This makes the table generation process highly scalable and flamelet libraries can be generated with significantly lower wall clock times. The model framework can be extended to varying pressures by including pressure as an additional manifold dimension. As this spray flame simulation is a constant pressure case, a $4 \mathrm{D}$ manifold has been used. The CFD solver calculates the mixture fraction $(\widetilde{Z})$, mixture fraction variance $\left(\widetilde{Z^{\prime \prime 2}}\right)$ and enthalpy for each cell. Mass-weighted fractions of the fuel that are injected are tracked

using marker Eq. 15 . At every time-step iteration, $\chi_{s t}$ values for a flamelet, $\widetilde{Z}, \widetilde{Z^{\prime \prime 2}}$ and time $t$ are passed to the tabulated model. A $4 \mathrm{D}$ interpolation scheme is used to calculate the species mass fractions from the chemistry tabulation instead of solving the flamelet equations. These species mass fractions are then used to calculate the temperature at each cell. For cases with multiple flamelets these steps are repeated for each flamelet and the species at each cell is given by Eq. (18). This tabulated model replaces the online flamelet calculation of the RIF model with data retrieval from a table. However, this has an impact on the physics of the solution. This model is now based on a steady SDR assumption and can no longer account for unsteady effects. It is not possible to generate a chemistry tabulation without the steady SDR assumption. The evolution of the scalar dissipation rate in time will vary depending on the flow conditions and the exact nature cannot be determined a priori. This has been the main factor that prevents tabulated models to account for history effects. Recently, the TFM approach i.e. tabulation without the history effects, has also been applied to Spray A cases with LES turbulence model by Ameen et al. [43.

\subsection{Tabulated Equivalent SDR Flamelet model (TESF)}

A model capable of including history effects was developed for 1D flamelets. The 1D simulations show that the unsteady effects are functions of $\chi_{s t}$ and $\mathrm{d} \chi_{s t} / \mathrm{d} t$. The secondary table is a $2 \mathrm{D}$ table where the correction factor $c$ is stored as a function of $\chi_{s t}$ and $\mathrm{d} \chi_{s t} / \mathrm{d} t$. The tabulation scheme's coupling with the 3D CFD solver is discussed in Fig. 9 For simulations with multiple flamelets, each flamelet has its own stoichiometric scalar dissipation rate and its history. At any given time-step the tabulated combustion model calculates the species based on the mixture fraction, mixture fraction variance, scalar dissipation rate and time. In this case, the correction factor $c$ is interpolated from the secondary 2D table. Using this value of $c$ and Eq. (4), an equivalent stoichiometric scalar dissipation rate is calculated from the temporal history of $\chi_{s t}$. This equivalent $\chi$, is used to retrieve the species from the multidimensional chemistry tabulation. At every time step for every flamelet, the value of $c$ is calculated based on $\chi_{s t}$ 
and $\mathrm{d} \chi_{s t} / \mathrm{d} t$. This implementation is shown in the schematic in Fig. 9 for a single flamelet. For multiple flamelets, the same procedure is repeated for each flamelet in an iterative manner.

\subsection{Results from $3 D$ spray simulations}

The baseline Spray A conditions are first studied using the three models. The injection duration is $1.5 \mathrm{~ms}$. During this injection process the flamelets experience rapid changes in scalar dissipation rates. The CFD model with the RIF combustion model has been extensively validated with the different mesh resolutions in our previous study [14]. A grid convergent mesh with smallest grid size of $0.25 \mathrm{~mm}$, established in our previous study [14] was used for all the three models.

Figure 10 shows the temporal evolution of the maximum temperature in the domain for the baseline Spray A case for the three models. Figure 11 shows the lift-off height corresponding to the results in Fig. 10. The results show that the TFM predicts lower ignition delay resulting in lower lift-off lengths as well compared to the RIF model. The lift-off length is defined as the minimum distance of the maximum $14 \% \mathrm{OH}$ contour from the nozzle [32. TFM under-predicts the ignition delay compared to RIF, which is similar to the 1D flamelet results. The TFM cannot account for the unsteady effects experienced by the flamelets, hence, as soon as the SDR goes below the quenching limit and after the ignition delay time has passed, it has a tendency to ignite. The RIF model is sensitive to the histories experienced by the flamelet as the flamelet equations are integrated over the varying SDRs. The implementation of the equivalent SDR model in the 3D CFD code (TESF) we observe that the autoignition predictions from the model are closer to the predictions of the RIF model. These observations in ignition are similar to the results from the 1D flamelets.

The qualitative differences due to history effects on the spray flame are demonstrated through the temperature contours shown in Fig. 12. During the transient flame development, it is observed that the TFM model ignites earlier with a high temperature region at $0.5 \mathrm{~ms}$ and reaches the quasi-steady state earlier compared to the RIF model. The RIF model at this stage predicts an unburnt solution. The qualitative predictions from the TESF model are similar to the predictions by the RIF model. The flame reaches a quasi-steady behavior at $1 \mathrm{~ms}$ and all three models predict similar temperature contours by this time. Thus, the TESF model is sensitive to the history effects for 3D spray simulations.

To further validate the TESF model, additional CFD simulations are carried out by varying the injection pressure of the spray from $50 \mathrm{MPa}$ to $150 \mathrm{MPa}$. Figure 13 shows ignition delay and lift-off lengths for injection pressure of the spray varied from $50 \mathrm{MPa}$ to $150 \mathrm{MPa}$. All other modeling parameters including mesh and spray constants are kept constant between the different combustion models. The tabulated model consistently predict lower ignition delay and lift-off lengths across the pressure sweep. The TESF model predicts ignition delays and lift-off lengths closer to the values predicted by the RIF model. This shows an improvement across a range of conditions. 


\subsection{Computational cost}

The RIF model incorporates the relevant physics to include the effects of unsteady strain rates experienced by a turbulent flame. However, the need for multiple flamelets and unsteady in situ solver and presumed PDF integration at each cell leads to very high computational costs. The RIF model simulation with 20 flamelets and $100 \mathrm{MPa}$ injection pressure required a total of $1780 \mathrm{CPU}$ hours. The corresponding case run with the TESF model required a total of $240 \mathrm{CPU}$ hours, which is a reduction of almost 7.5 times. Similar speed-ups were observed for other cases. The $50 \mathrm{MPa}$ injection pressure case with 20 flamelets and RIF model required a total of 1382 CPU hours and the same run with the TESF model required 153.6 CPU hours. These results show that tabulated models can effectively reduce computational costs while maintaining the same level of modeling accuracy. The comparisons presented here are for simulations run with 32 processors in parallel on the same high performance cluster with $2.6 \mathrm{GHz}$ Pentium Xeon processors. Future work would aim at extending such high fidelity combustion modeling techniques to LES approach where computational cost has been a major challenge. A potential saving associated with the tabulation of unsteady SDR effects can be significant. The total cost of generating the table was approximately 8 CPU hours. Due to the nature of the problem, the table generation code could be parallelized using the Message Passing Interface (MPI) framework on a large number of processors and completed in a few minutes. The difference in the memory requirement of the TESF and TFM tabulation is negligible. In this version of the CFD code, the Message Passing Interface framework was used for parallelization. Each processor (rank) has its own memory and its copy of the tabulation.

\section{Conclusions}

The history effects due to unsteady SDR of flamlets at high pressure and temperature conditions have significant impact on autoignition and flame lift-off at diesel engine conditions. These effects are demonstrated through various modeling approaches on 1D flamelets. Results from 1D unsteady flamelet models, with a 106 species n-dodecane mechanism were compared to steady SDR assumption models. The difference in autoignition due to history effects were found to be directly proportional to the magnitude of the temporal gradient of $\chi_{s t}$ of a flamelet. The history effects become negligible for cases where temporal gradients are low. Tabulated flamelet models used for high temperature and pressure simulations need to incorporate such physics in order to predict autoignition and flame stabilization accurately. However, in situ models like RIF, that account for such effects are computationally expensive.

A formulation that captures unsteady history effects in tabulated combustion models is proposed and validated in $1 \mathrm{D}$ flamelets. This involves calculating a weighted average of the stoichiometric scalar dissipation rate over the flamelet history in which higher weights are assigned for the most recent histories. This modeling approach is first tested for 1D CFDF problems with non-uniformly and arbitrarily varying $\chi_{s t}$. A non-uniform $\chi_{s t}$ variation, similar to a spray flame, is imposed upon a 1D flamelet. The steady strain assumption under-predicts autoignition compared to the in-situ RIF solver. The equivalent SDR concept predicts both stages of ignition in tune with the unsteady in 
situ flamelet solver.

This equivalent SDR concept was extended to modeling of a 3D spray flame within a RANS framework. The observations with respect to autoignition are similar to the $1 \mathrm{D}$ flamelet simulations. Three different combustion models were compared: RIF, TFM, and TESF. The tabulated models (TFM and TESF) are based on the structure of the multi-flamelet RIF model by replacing the flamelet equation solver by a novel 4D tabulation technique without progress variables. The TFM with steady strain assumption, underpredicts ignition delay and lift-off compared to the RIF model. The TESF model on the other hand accounts for history effects in 3D spray combustion simulations and predicts autoignition and flame lift-off length close to the ones predicted by the RIF model. The results across a range of injection pressures show consistent improvement over predictions from tabulated models with steady strain rate assumptions. The equivalent SDR model (TESF) is a promising strategy to capture unsteady effects in CFD simulations without resorting to in situ unsteady flamelet calculations with detailed chemistry.

\section{Acknowledgment}

The submitted manuscript has been created by UChicago Argonne, LLC, Operator of Argonne National Laboratory (Argonne). Argonne, a U.S. Department of Energy Office of Science laboratory, is operated under Contract No. DE-AC02-06CH11357. The U.S. Government retains for itself, and others acting on its behalf, a paid-up nonexclusive, irrevocable worldwide license in said article to reproduce, prepare derivative works, distribute copies to the public, and perform publicly and display publicly, by or on behalf of the Government. The research was funded by DOEs Office of Vehicle Technologies, Office of Energy Efficiency and Renewable Energy under Contract No. DEAC02-06CH11357. The authors wish to acknowledge the computational resources of 'Fusion' and 'Blues' clusters at Argonne National Laboratory.

\section{References}

[1] N. Peters, F. A. Williams, Liftoff characteristics of turbulent jet diffusion flames, AIAA Journal 21 (3) (1983) $423-429$.

[2] E. Mastorakos, A. Taylor, J. Whitelaw, Scalar dissipation rate at the extinction of turbulent counterflow nonpremixed flames, Combust. Flame 91 (1) (1992) 55-64.

[3] F. N. Egolfopoulos, C. S. Campbell, Unsteady counterflowing strained diffusion flames: diffusion-limited frequency response, Journal of Fluid Mechanics 318 (1996) 1-29.

[4] J. Kistler, C. Sung, T. Kreut, C. Law, M. Nishioka, Extinction of counterflow diffusion flames under velocity oscillations, Symp.(Int.) Combust 26 (1996) 113-120.

[5] H. Im, J. Bechtold, C. Law, Counterflow diffusion flames with unsteady strain rates, Combustion Science and Technology 106 (4-6) (1995) 345-361. 
[6] T. Brown, R. Pitz, C. Sung, Oscillatory stretch effects on the structure and extinction of counterflow diffusion flames, Symp. (Int.) Combust 27 (1998) 703-710.

[7] R. S. Barlow, J.-Y. Chen, On transient flamelets and their relationship to turbulent methane-air jet flames, Symp. (Int.) Combust. 24 (1992) 231-237.

[8] H. Pitsch, H. Barths, N. Peters, Three-dimensional modeling of nox and soot formation in di-diesel engines using detailed chemistry based on the interactive flamelet approach, Tech. rep., SAE Technical Paper (1996).

[9] H. Barths, C. Hasse, G. Bikas, N. Peters, Simulation of combustion in direct injection diesel engines using a eulerian particle flamelet model, Symp. (Int.) Combust. 28 (2000) 1161-1168.

[10] H. Barths, N. Peters, N. Brehm, A. Mack, M. Pfitzner, V. Smiljanovski, Simulation of pollutant formation in a gas-turbine combustor using unsteady flamelets, Symp. (Int.) Combust. 27 (2) (1998) 1841-1847.

[11] T. Husberg, I. Denbratt, M. Ringvik, J. Engström, Heavy-duty diesel combustion with ultra-low nox and soot emissions-a comparison between experimental data and cfd simulations, Tech. rep., SAE Technical Paper (2005).

[12] S. Singh, R. D. Reitz, M. P. Musculus, Comparison of the characteristic time (ctc), representative interactive flamelet (rif), and direct integration with detailed chemistry combustion models against optical diagnostic data for multi-mode combustion in a heavy-duty di diesel engine, Tech. rep., SAE technical paper (2006).

[13] C. Hergart, H. Barths, N. Peters, Modeling the combustion in a small-bore diesel engine using a method based on representative interactive flamelets, Tech. rep., SAE Technical Paper (1999).

[14] P. Kundu, Y. Pei, M. Wang, R. Mandhapati, S. Som, Evaluation of turbulence-chemistry interaction under diesel engine conditions with multi-flamelet rif model, Atomization and Sprays 24 (2014).

[15] G. DErrico, T. Lucchini, F. Contino, M. Jangi, X.-S. Bai, Comparison of well-mixed and multiple representative interactive flamelet approaches for diesel spray combustion modelling, Combustion Theory and Modelling 18 (1) (2014) 65-88.

[16] P. Pal, S. Keum, H. G. Im, Assessment of flamelet versus multi-zone combustion modeling approaches for stratified-charge compression ignition engines, International Journal of Engine Research 17 (3) (2016) 280-290.

[17] M. Ihme, H. Pitsch, Prediction of extinction and reignition in nonpremixed turbulent flames using a flamelet/progress variable model: 2. Application in LES of Sandia flames D and E, Combust. Flame 155 (1) (2008) 90-107.

[18] C. D. Pierce, P. Moin, Progress-variable approach for large-eddy simulation of non-premixed turbulent combustion, Journal of Fluid Mechanics 504 (2004) 73-97. 
[19] M. Ihme, Y. C. See, Prediction of autoignition in a lifted methane/air flame using an unsteady flamelet/progress variable model, Combust. Flame 157 (10) (2010) 1850-1862.

[20] M. M. Ameen, J. Abraham, RANS and LES study of lift-off physics in reacting diesel jets, Tech. rep., SAE Technical Paper (2014).

[21] C. Bajaj, M. Ameen, J. Abraham, Evaluation of an unsteady flamelet progress variable model for autoignition and flame lift-off in diesel jets, Combustion Science and Technology 185 (3) (2013) 454-472.

[22] V. Oijen, L. D. Goey, Modelling of premixed laminar flames using flamelet-generated manifolds, Combustion Science and Technology 161 (1) (2000) 113-137.

[23] C. Bekdemir, L. Somers, L. de Goey, J. Tillou, C. Angelberger, Predicting diesel combustion characteristics with large-eddy simulations including tabulated chemical kinetics, Proc. Combust. Inst. 34 (2013) 3067-3074.

[24] F. Tap, P. Schapotschnikow, Efficient combustion modeling based on Tabkin $\AA$ CFD look-up tables: a case study of a lifted diesel spray flame, Tech. rep., SAE Technical Paper (2012).

[25] A. Wehrfritz, O. Kaario, V. Vuorinen, B. Somers, Large Eddy Simulation of n-dodecane spray flames using Flamelet Generated Manifolds, Combust. Flame 167 (2016) 113-131.

[26] M. J. McNenly, R. A. Whitesides, D. L. Flowers, Faster solvers for large kinetic mechanisms using adaptive preconditioners, Proc. Combust. Inst. 35 (2015) 581-587.

[27] D. Haworth, M. Drake, S. Pope, R. Blint, The importance of time-dependent flame structures in stretched laminar flamelet models for turbulent jet diffusion flames, Symp. (Int.) Combust. 22 (1989) 589-597.

[28] B. Cuenot, F. N. Egolfopoulos, T. Poinsot, An unsteady laminar flamelet model for non-premixed combustion, Combustion Theory and Modelling 4 (2000) 77-97.

[29] S. Delhaye, L. Somers, J. Van Oijen, L. De Goey, Incorporating unsteady flow-effects in flamelet-generated manifolds, Combust. Flame 155 (2008) 133-144.

[30] G. P. Smith, D. M. Golden, M. Frenklach, N. W. Moriarty, B. Eiteneer, M. Goldenberg, C. T. Bowman, R. K. Hanson, S. Song, W. J. Gardiner, et al., Gri 3.0, Gas Research Institute, Chicago, IL, http://www. me. berkeley. edu/gri_mech.

[31] S. Delhaye, L. Somers, J. Van Oijen, L. de Goey, Incorporating unsteady flow-effects beyond the extinction limit in flamelet-generated manifolds, Proc. Combust. Inst. 32 (2009) 1051-1058.

[32] Engine Combustion Network http://www.sandia.gov/ecn/, Tech. rep., Sandia National Laboratory (2013). 
[33] Z. Luo, S. Som, S. M. Sarathy, M. Plomer, W. J. Pitz, D. E. Longman, T. Lu, Development and validation of an n-dodecane skeletal mechanism for spray combustion applications, Combustion Theory and Modelling 18 (2) (2014) 187-203.

[34] L. M. Pickett, C. L. Genzale, G. Bruneaux, L.-M. Malbec, L. Hermant, C. A. Christiansen, J. Schramm, Comparison of diesel spray combustion in different high-temperature, high-pressure facilities, SAE International Journal of Engines 3 (2) (2010) 156-181.

[35] K. Richards, P. Senecal, E. Pomraning, Converge (version 2.1. 0) theory manual, Convergent Science, Inc., Middleton, WI.

[36] Z. Han, R. D. Reitz, Turbulence modeling of internal combustion engines using RNG $\kappa-\varepsilon$ models, Combustion Science and Technology 106 (4-6) (1995) 267-295.

[37] R. D. Reitz, R. Diwakar, Structure of high-pressure fuel sprays, Tech. rep., SAE Technical Paper (1987).

[38] R. D. Reitz, Modeling atomization processes in high-pressure vaporizing sprays, Atomisation Spray Technology 3 (1987) 309-337.

[39] M. A. Patterson, R. D. Reitz, Modeling the effects of fuel spray characteristics on diesel engine combustion and emission, Tech. rep., SAE Technical Paper (1998).

[40] N. Frossling, Evaporation, heat transfer, and velocity distribution in two-dimensional and rotationally symmetrical laminar boundary-layer flow, Tech. rep., DTIC Document (1956).

[41] A. B. Liu, D. Mather, R. D. Reitz, Modeling the effects of drop drag and breakup on fuel sprays, Tech. rep., DTIC Document (1993).

[42] N. Peters, Laminar diffusion flamelet models in non-premixed turbulent combustion, Progress in Energy and Combustion Science 10 (3) (1984) 319-339.

[43] M. M. Ameen, P. Kundu, S. Som, Novel tabulated combustion model approach for lifted spray flames with large eddy simulations, SAE International Journal of Engines 9 (2016-01-2194). 


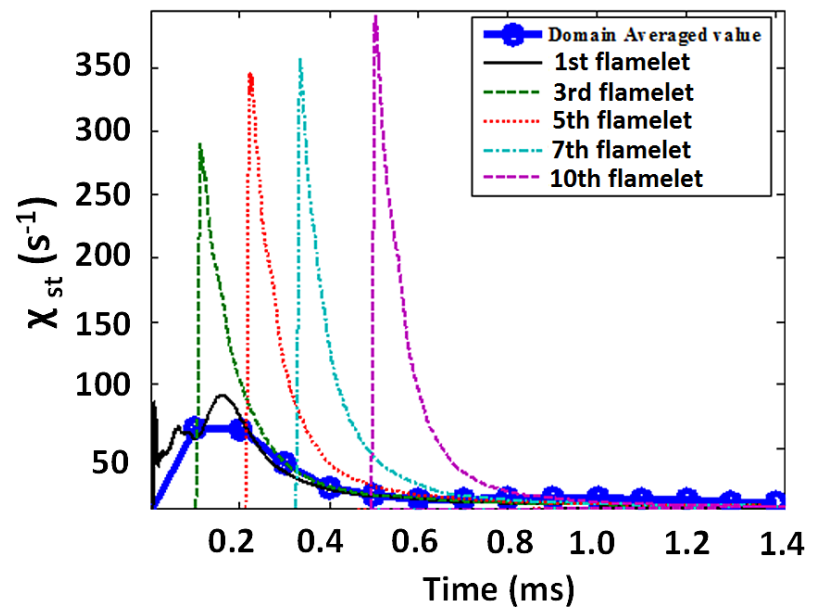

Figure 1: Scalar dissipation rates experienced by flamelets for diesel fuel spray injection.
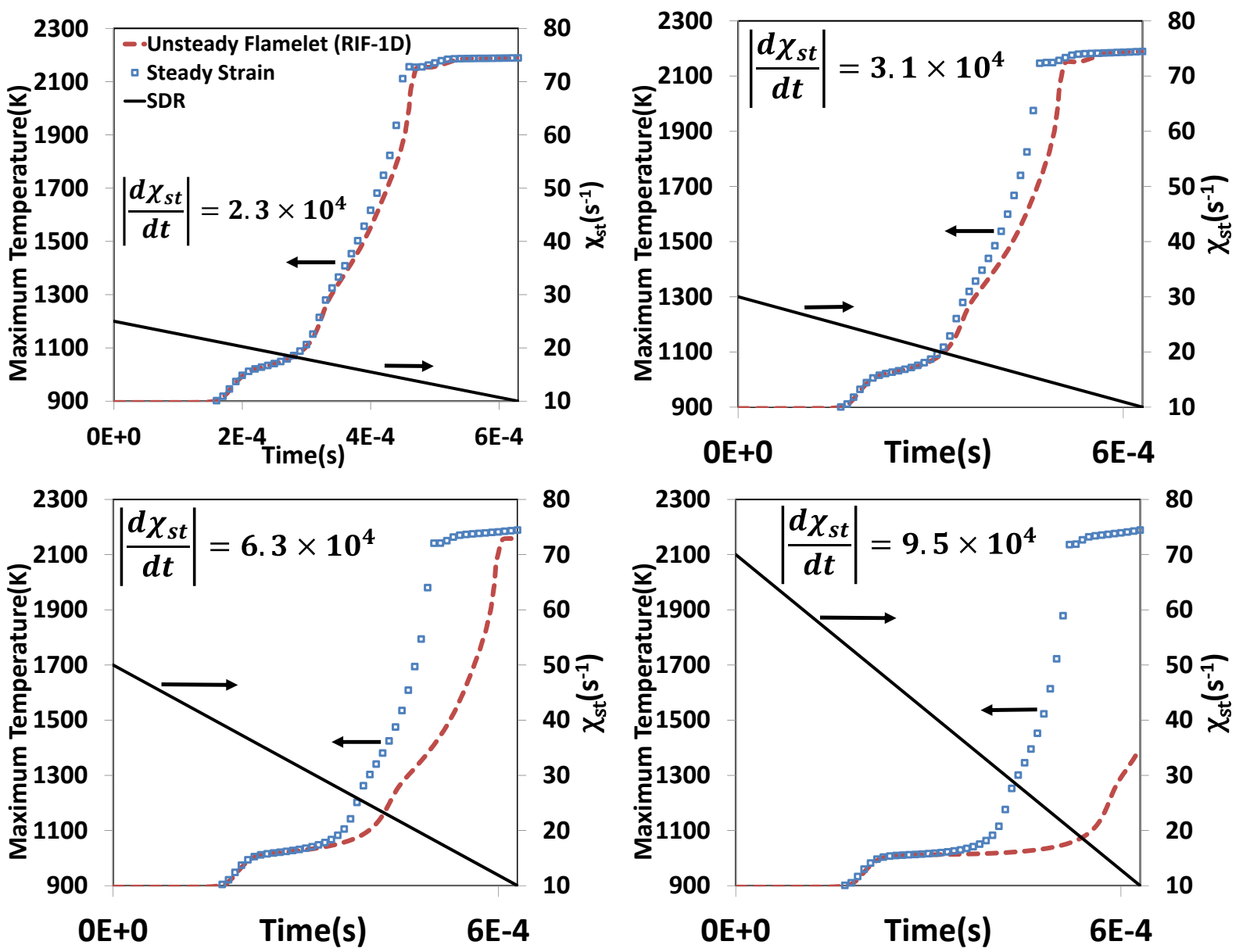

Figure 2: Autoignition of flamelets under varying scalar dissipation rates for different imposed gradients. Flamelet RIF-1D is shown in red lines; steady SDR model, which is equivalent to a tabulated flamelet is shown by blue symbols. The imposed $\chi_{s t}$ as a function of time is shown by the black solid lines. 


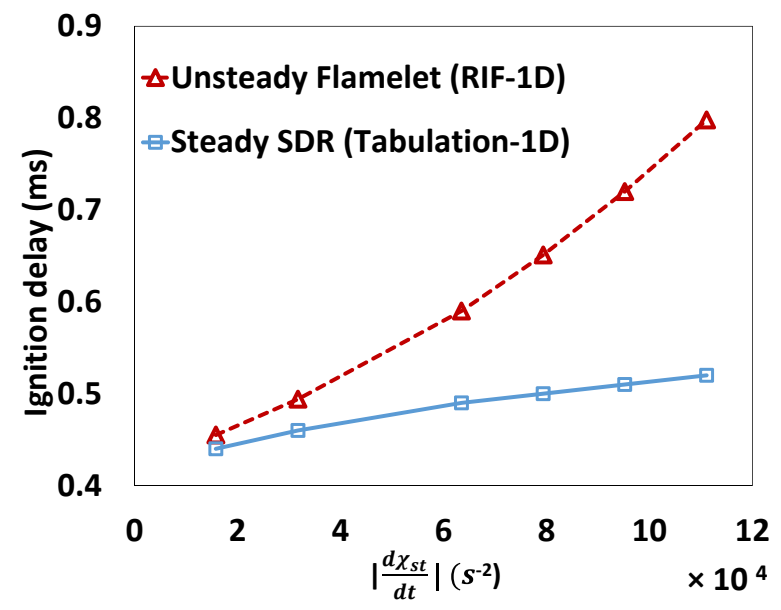

Figure 3: Ignition delays for flamelets with different temporal gradients in $\chi_{s t}$ as a function of $\left|\frac{d \chi_{s t}}{d t}\right|$. The steady SDR assumption leads to a longer ignition delay than the RIF-1D model. The differences due to history effects become significant for larger gradients.

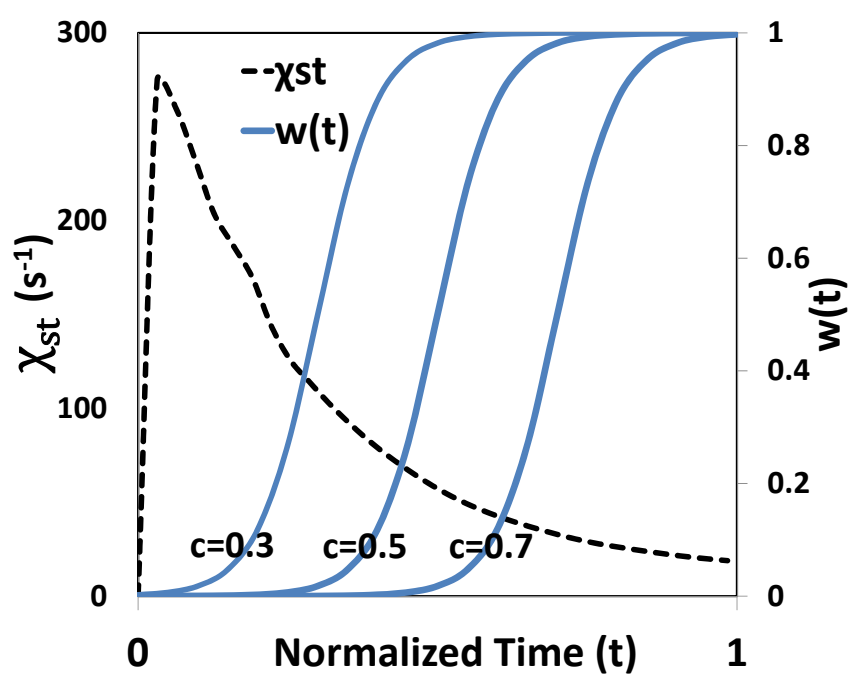

Figure 4: Weight function on secondary y-axis (right) along with the flamelet SDR history plotted with respect to non-dimensional time, for different values of the parameter $c$. At each time-step, the weight function is used to calculated a weighted average SDR over the flamelet's history. 

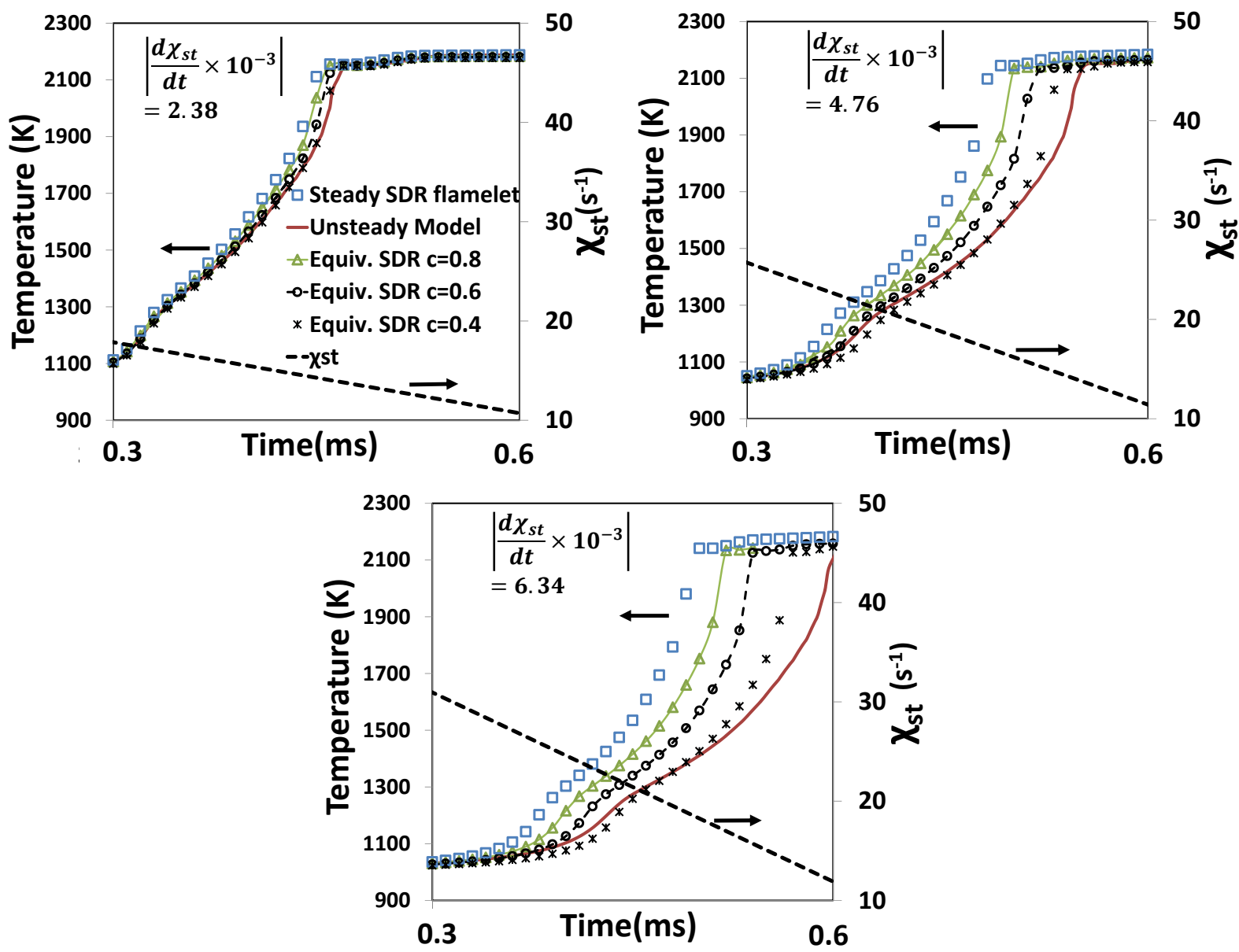

Figure 5: Autoignition of flamelets under varying scalar dissipation rates are shown for different imposed gradients. The unsteady flamelet (RIF-1D) and steady SDR models are shown by solid red lines and blue symbols respectively. The equivalent SDR method applied to the steady strain assumption are shown with the green triangles, black circles and cross symbols for different variations of $c$.

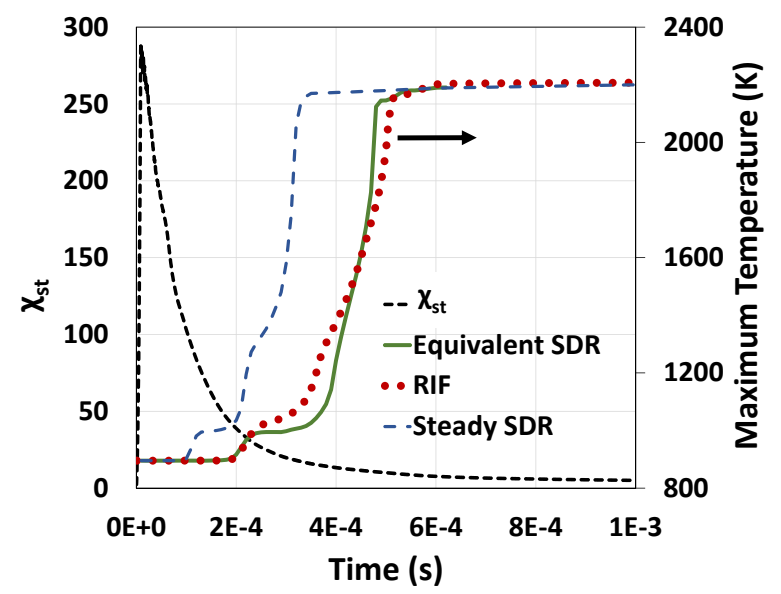

Figure 6: Ignition of a 1D flamelet with non-linearly varying SDR imposed from a typical spray flame condition shown on the primary y-axis (left). The maximum temperatures with respect to time are shown with three different flamelet models: 1) Equivalent SDR (solid green), 2) RIF (dots red), 3) Steady SDR (dashed blue). 


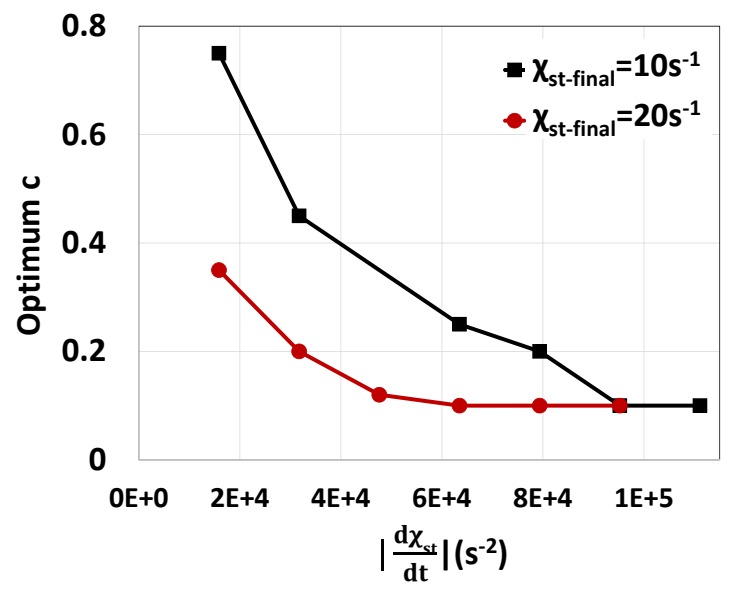

Figure 7: Correction factor $c$ as a function of $d \chi_{s t} / d t$ for different $\chi_{s t}$

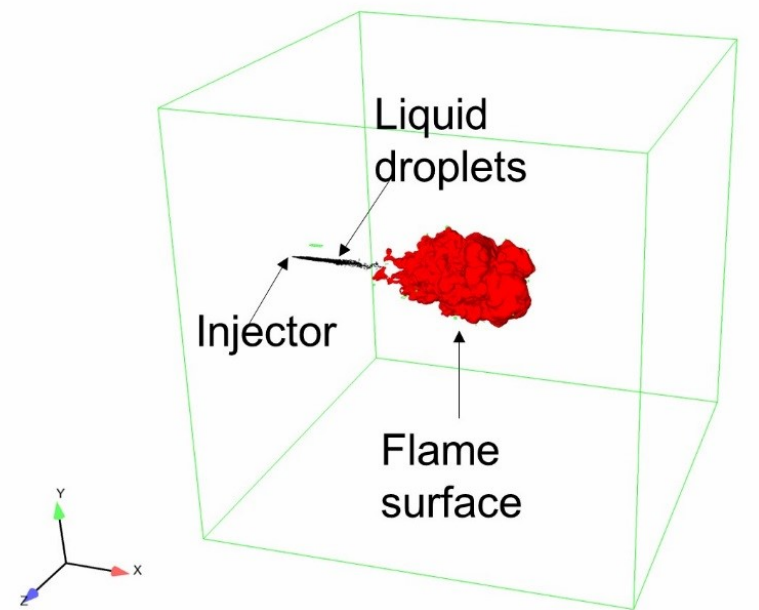

Figure 8: Computational domain showing injected liquid droplets and flame surface. 
RIF

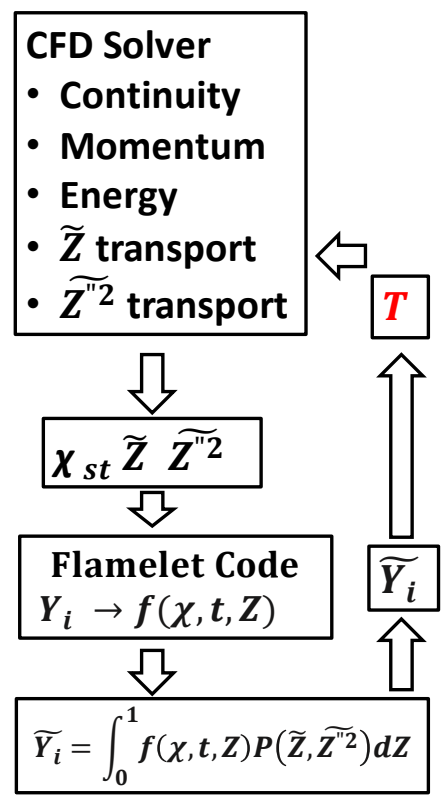

TFM

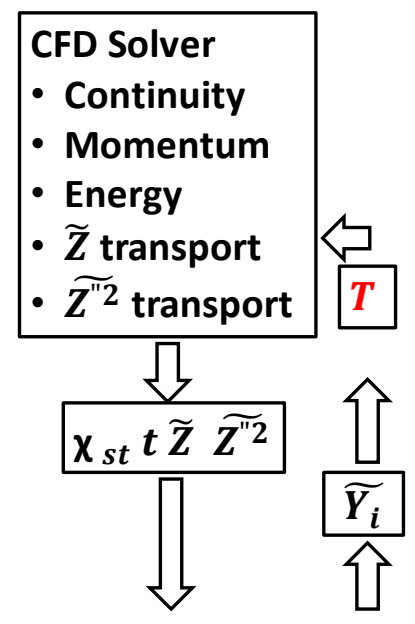

\begin{tabular}{|l|}
\hline 4D Interpolator \\
$\widetilde{Y}_{i} \rightarrow \boldsymbol{f}\left(\chi_{s t}, \mathrm{t}, \widetilde{Z} \underset{\left.Z^{\prime 2}\right)}{|c|}\right.$ \\
\hline Chemistry Table \\
\hline
\end{tabular}
TESF

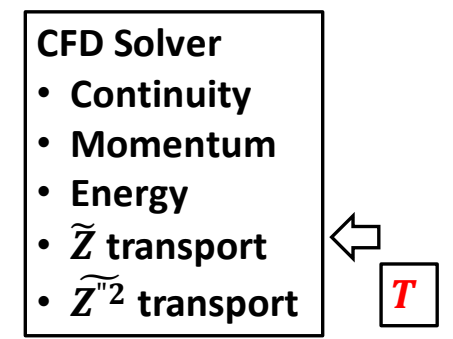

\begin{tabular}{|c|c|}
\hline$\chi_{s t}(t) \frac{\mathrm{d} \chi_{s t}}{d t} t \widetilde{Z} \widetilde{Z^{\prime 2}}$ & $\widehat{U}$ \\
\hline$\chi_{c}=f\left(\frac{\mathrm{d} \chi_{s t}}{d t}, \chi_{s t}(t)\right)$ & $\widetilde{Y_{i}}$ \\
\hline
\end{tabular}

4D Interpolator

$\widetilde{Y_{i}} \rightarrow \boldsymbol{f}\left(\chi_{c}, \mathrm{t}, \widetilde{Z} \overline{Z^{\prime 2}}\right)$

Chemistry Table

Figure 9: Schematic showing the implementation of the RIF, TFM and TESF models.

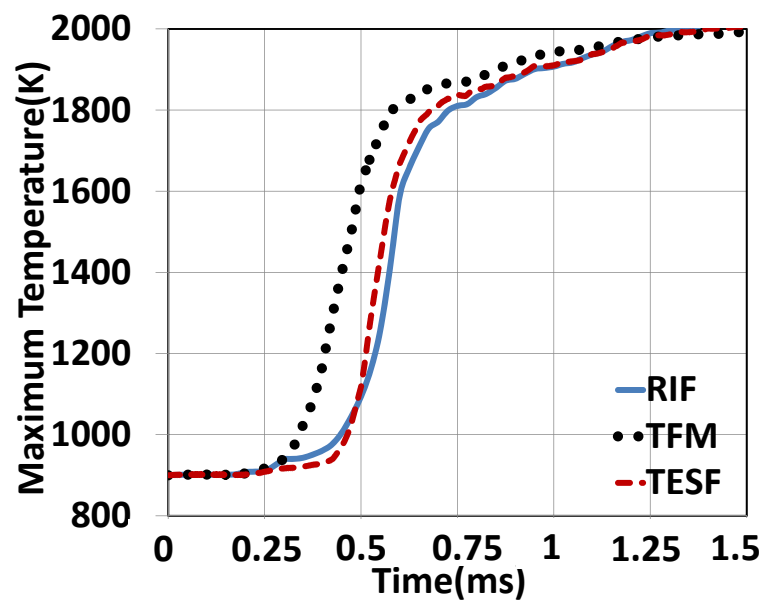

Figure 10: Temporal evolution of maximum temperature in the domain compared for RIF, TFM and TESF models under Spray A flame conditions. 


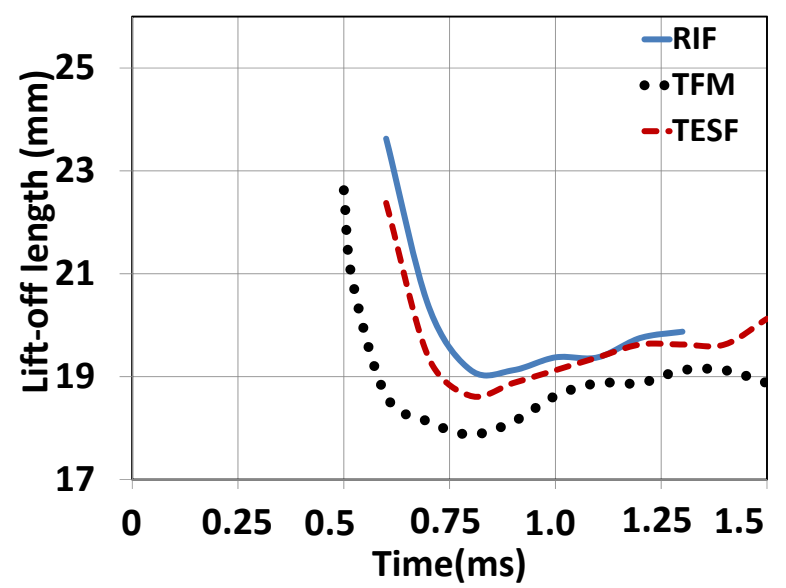

Figure 11: Lift-off lengths predicted by RIF , TFM and TESF models for the Spray A flame conditions.
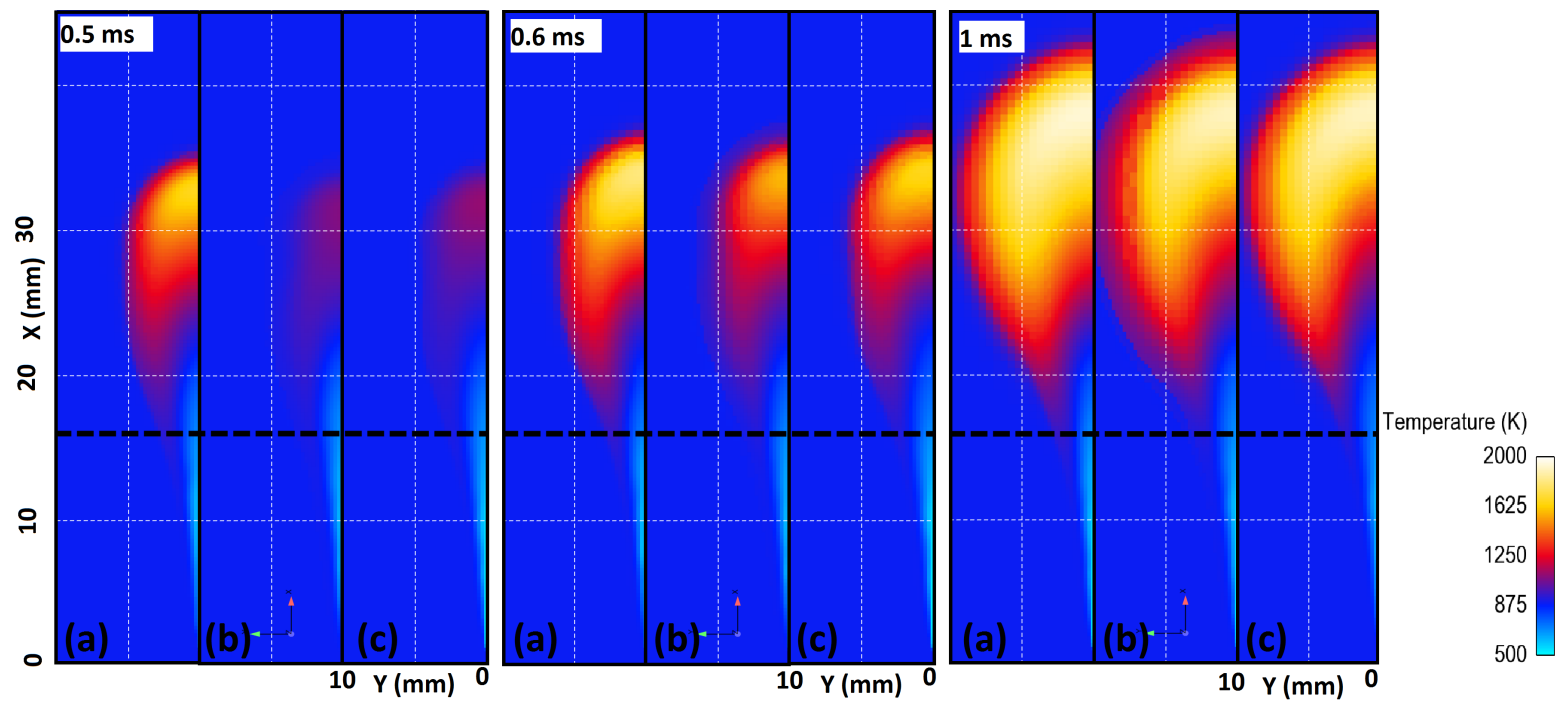

Figure 12: Cut-planes showing temperature contours at different time step with (a)TFM (b)RIF (c)TESF for the Spray A flame simulation. The experimental lift-off length is represented in by the black dotted line.
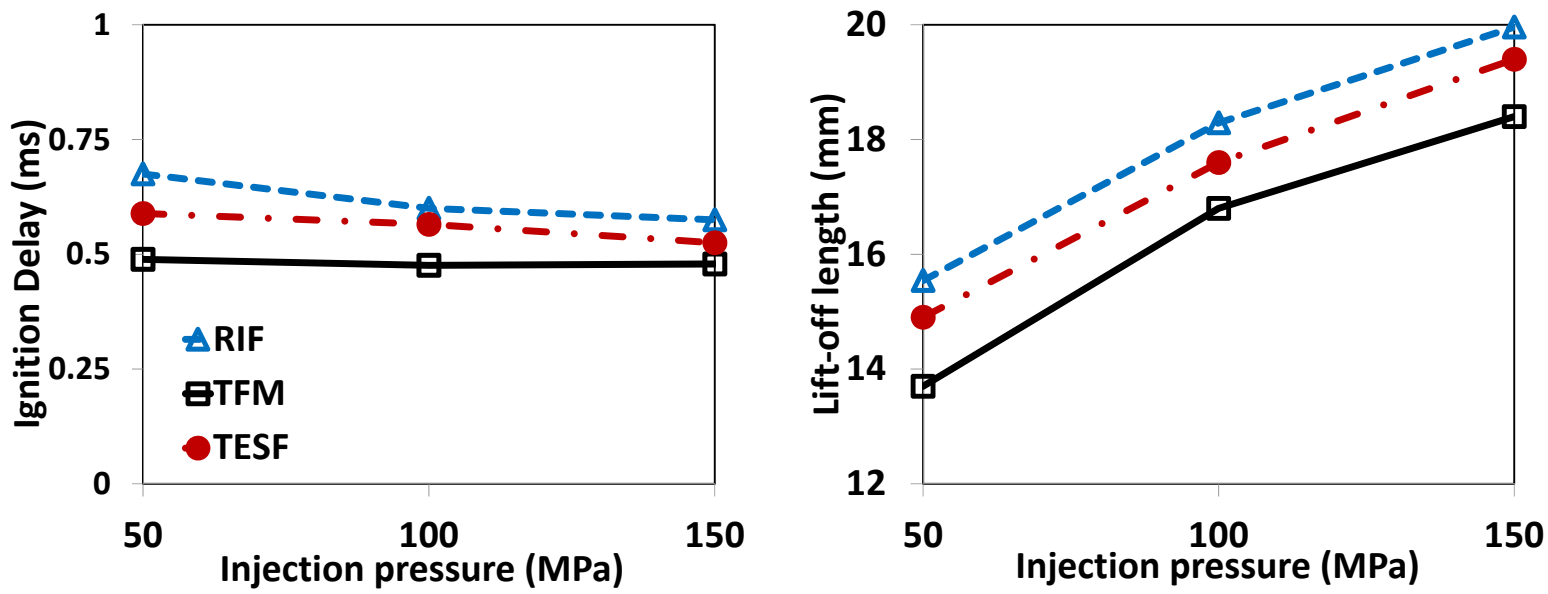

Figure 13: Ignition delay and lift-off lengths predicted by RIF, TFM and TESF models across different injection pressures for spray flame simulations. 